\title{
The private sector as a provider of family planning services in Egypt: Challenges and opportunities [Arabic]
}

\author{
Nahla G. Abdel-Tawab \\ Population Council \\ Doaa Oraby \\ Population Council \\ Benjamin Bellows \\ Population Council
}

Follow this and additional works at: https://knowledgecommons.popcouncil.org/departments_sbsr-rh

Part of the Demography, Population, and Ecology Commons, Family, Life Course, and Society Commons, and the International Public Health Commons How does access to this work benefit you? Let us know!

\section{Recommended Citation}

Abdel-Tawab, Nahla G., Doaa Oraby, and Benjamin Bellows. 2016. "The private sector as a provider of family planning services in Egypt: Challenges and opportunities [Arabic]," Policy brief. Cairo: Population Council. 



\section{شكل ا : مصدر الحصول على وسيلة تنظيم الأسرة $(r+1 \leq-1990)$}

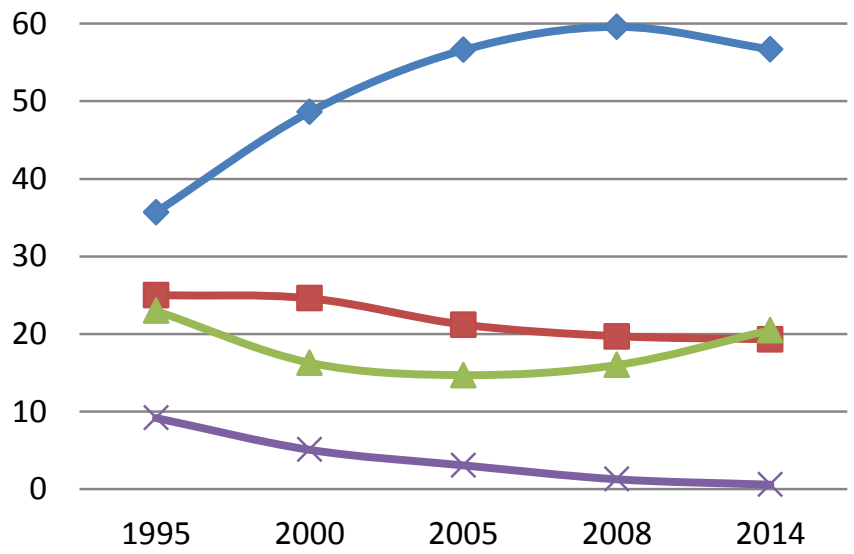

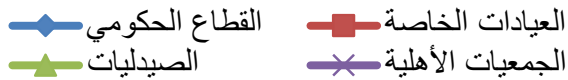

ويستعرض هذا الملخص الوضع الحالى للقطاع الخاص في تقديم

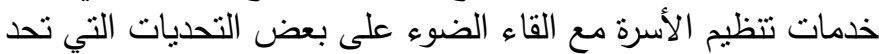

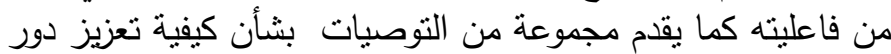

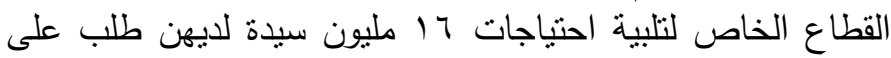

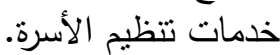

\section{مصادر الأبانأنات}

\section{تستند النتائج والتوصيات الواردة في هذا الملخص إلى:}

(1) مراجعة المقالات العلمية والتقارير التي صدرت في الفترة بين

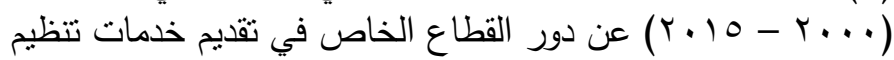

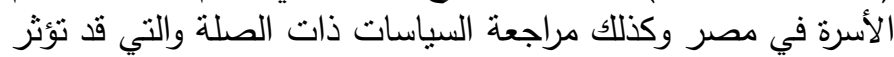

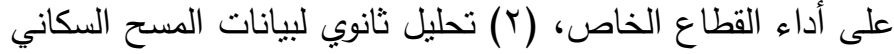

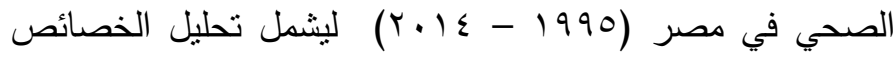
الاجتماعية والديموجرافية والاقتصادية للسيدات اللاتي يمكن استهدافهن

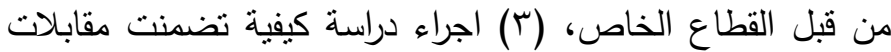

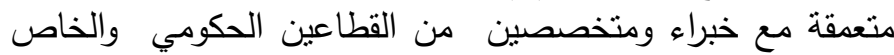
وبعض الهيئات الدولية، ومقابلات متعدقة مع مقدمي الخدمة بالقطاع

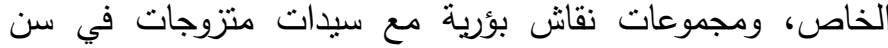
الإنجاب (11 (1- •ـ سنة) ممن سبق لهن استخدام وسائل نتظيم الأسرة

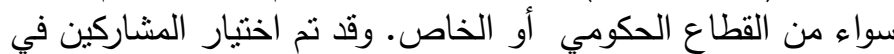
الدراسة من محافظات القاهرة وأسيوط والغربية.

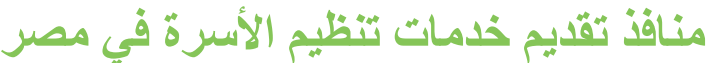

تقدم خدمات تتظيم الأسرة من خلال شبكة واسعة من المراكز الحكومية

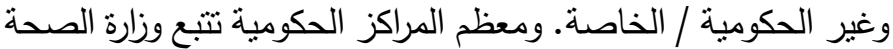
والسكان وتشمل مراكز الرعاية الصحية الأولية، في حين تشمل المراكز
منذ منتصف الستنيات من القرن الماضي ومع انطلاق البرنامج

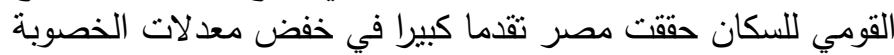

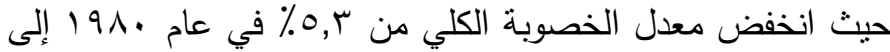

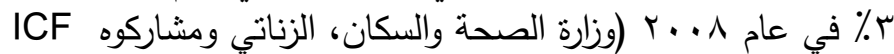

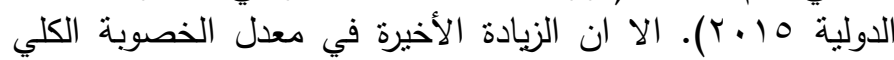

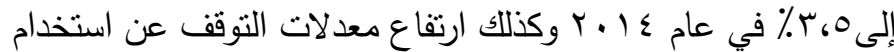

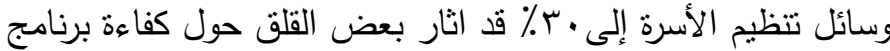

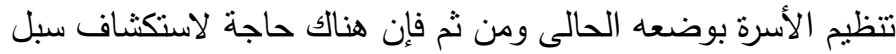

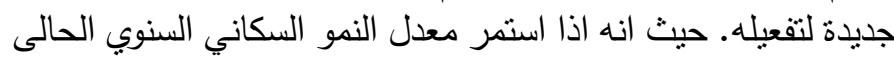

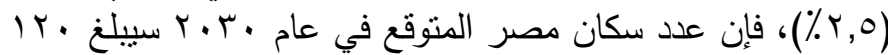

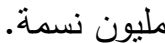

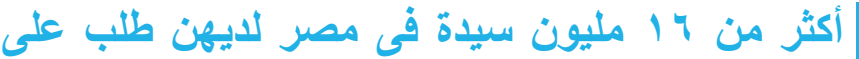 خدمات تنظيم الأسرة.}

يوجد حاليا أكثر من 17 مليون سيدة في مصر لديها طلب على الإنى

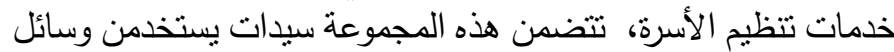

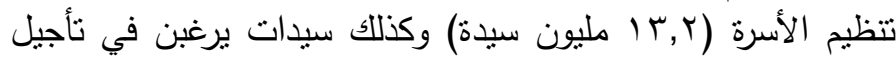
الحمل أو التوقف عن الانجاب ولكن لا يستخدمن وسيلة من وسائل تتظيم الأسرة (r,^ مليون سيدة).

وعلى الرغم من أن وزارة الصحة والسكان هي المصدر الرئيسي لخدمات تتظيم الأسرة، الا أنها قد لا تكون قادرة على التئ تلبية احتباجات

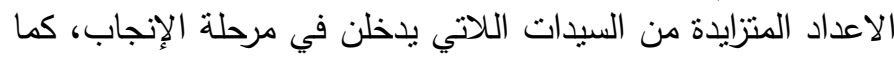

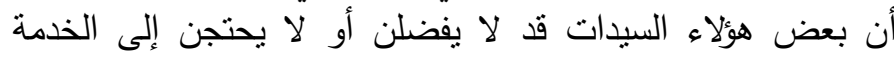

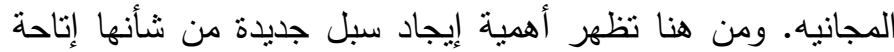
الفرصة امام القطاع الخاص (سواء الهادف للربح أو غير الهاد الهادف للربح) للمشاركة في تقديم خدمات تنظيم الأسرة.

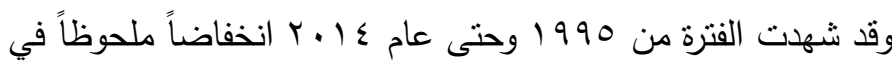

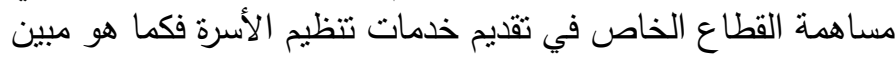

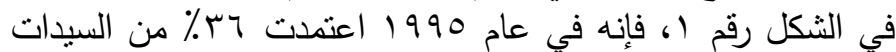

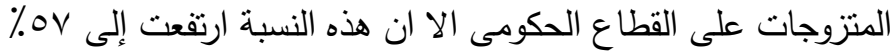

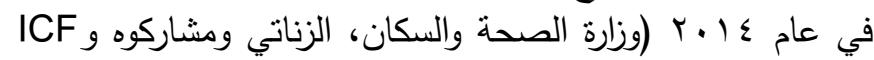

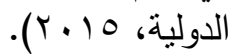

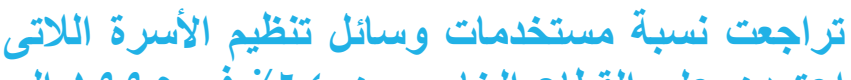

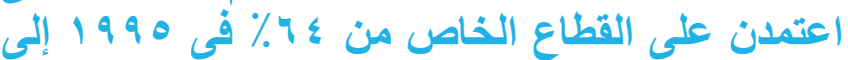

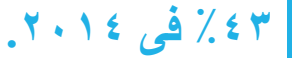

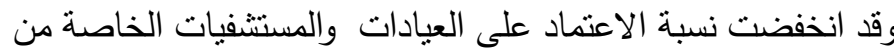

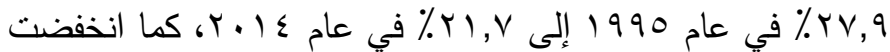

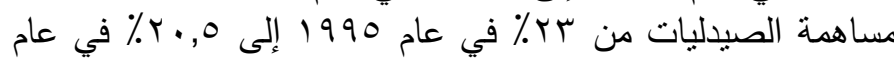

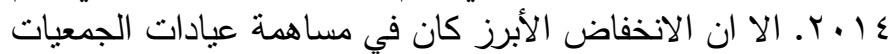

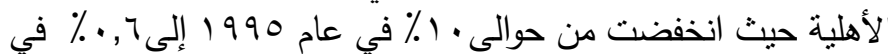

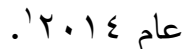




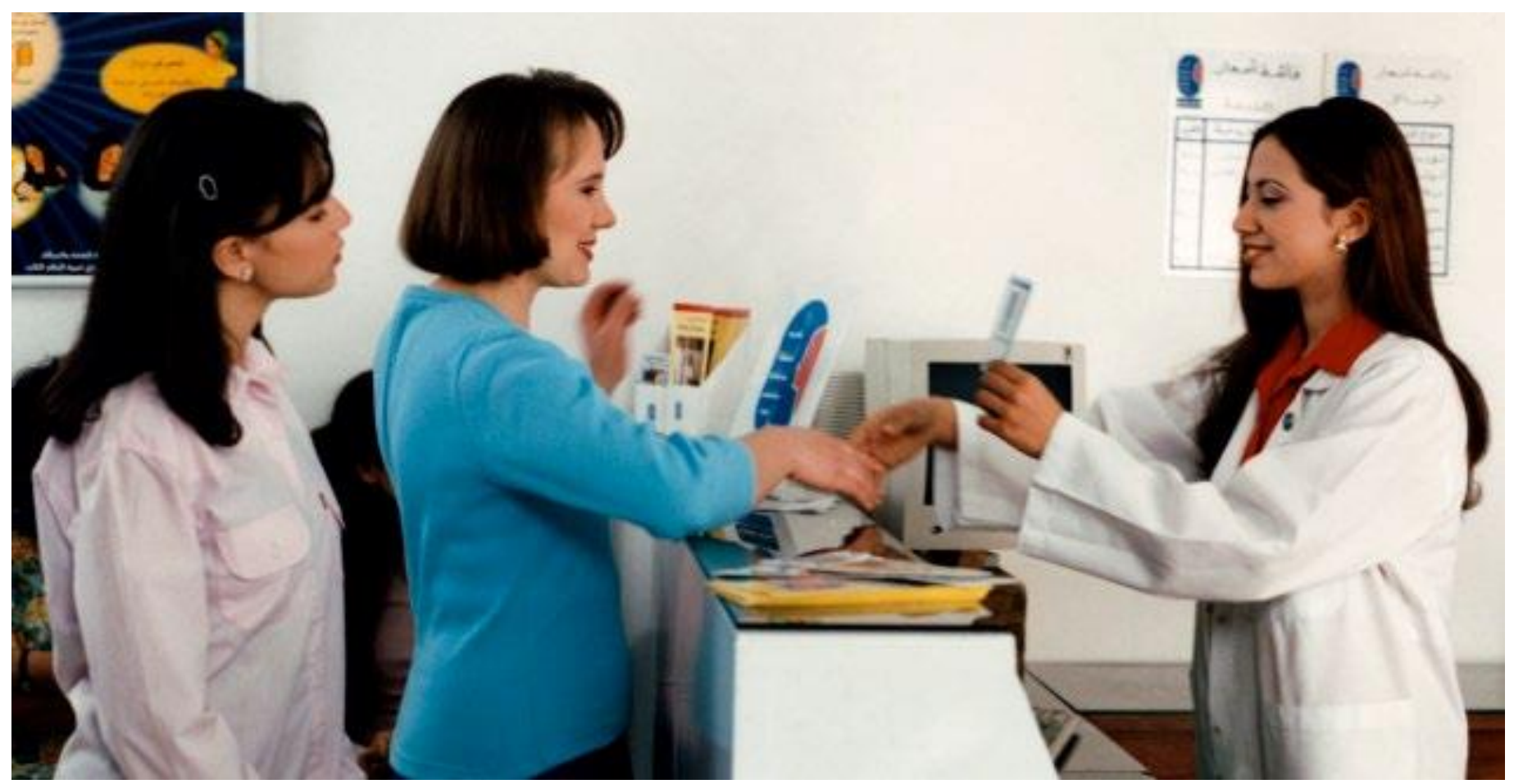

أما عن أطباء ومستشفيات القطاع الخاص فطبقاً لبيانات المسح

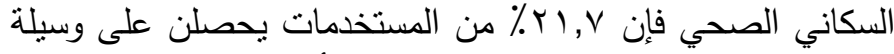

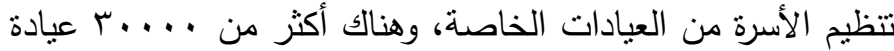

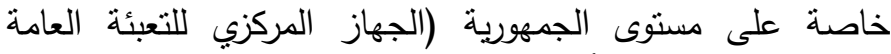

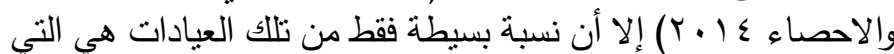

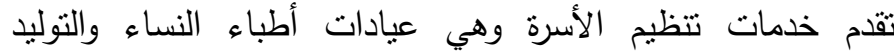

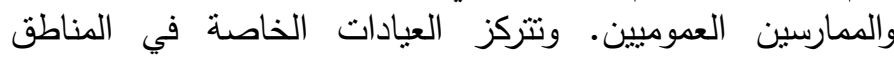

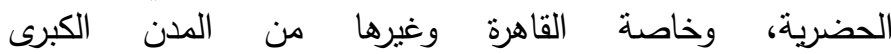
.(Nakhimovsky et al., 2011)

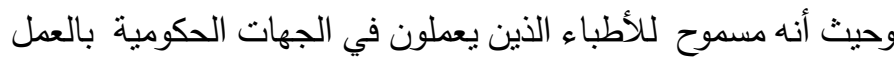

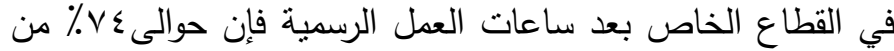

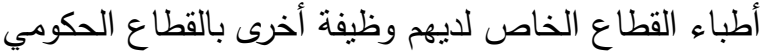

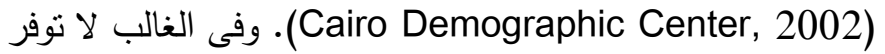

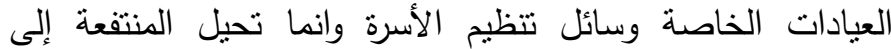

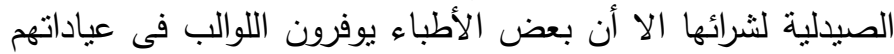

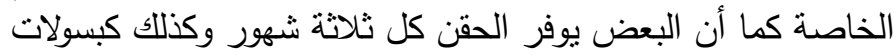

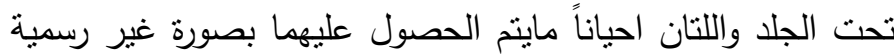
من القطاع الحكومى. وتتوقف أسعار خدمات تتظيم الأسرة التي تقدمها

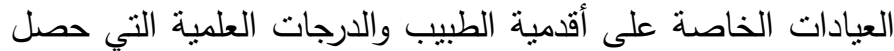

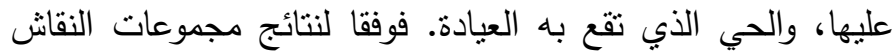
البؤرية مع السيدات والمقابلات مع أطباء القطاع الخاص والخيات الخبراء

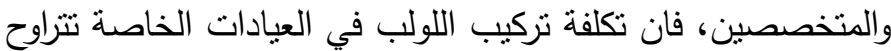

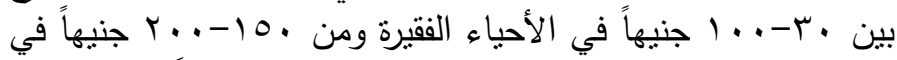

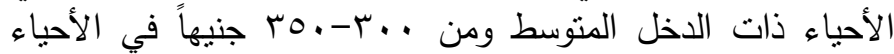

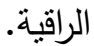

ومن ناحية اخرى فإن حوالى ب ٪ من مستخدمات وسائل تنظيم الأسرة

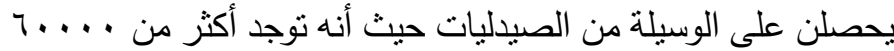
صيدلية خاصة موزعة على انحاء الجمهورية (الجهاز المركزي للتعبئة
غير الحكومية الهادفة للربح أطباء القطاع الخاص والصيدليات

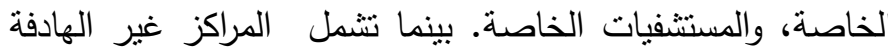

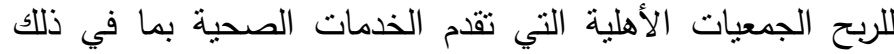

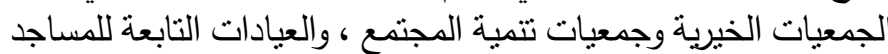

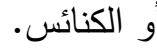

وتعد وزارة الصحة والسكان هى المصدر الرئيسي لخدمات تتظيم الأسرة

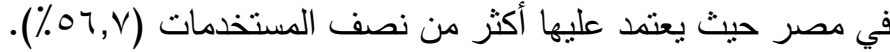
ويوجد في مصر ما يقرب من ل ...0 مركز تنظيم أسرة تابع لوزارة

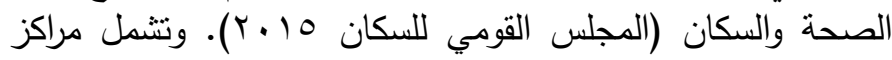

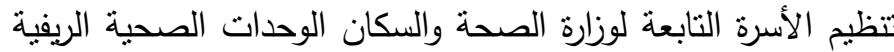

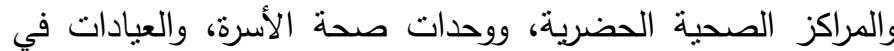

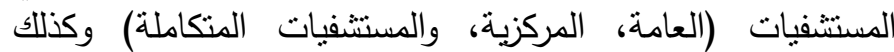
الوحدات المتتقلة. وتقدم مراكز وزارة الصحة والسكان خدان خدمات نتظيم

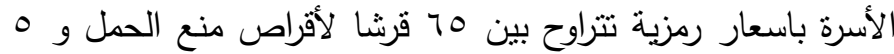
جنيهات للكبسولات تحت الجلدا. وتقدم الخدمات من الساعة التاعة الثامنة صباحا حتى الساعة الثانية ظهراً على مدار ستة أيام في الأسبوع.

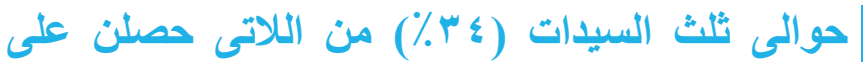

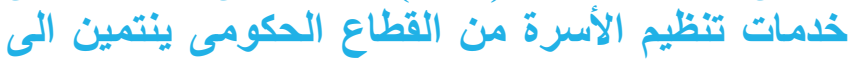

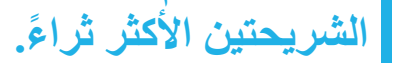

وتقدم مراكز وزارة الصحة والسكان وسائل تتظيم الأسرة التالية: اقراص

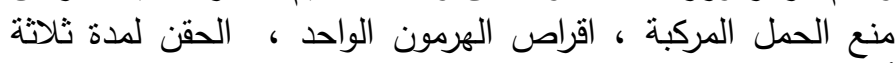

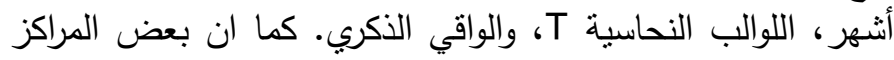

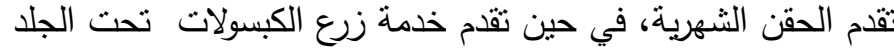

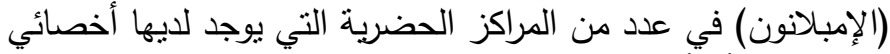
نساء وتوليد أو أخصائي مدرب في الصحة الإنجابية. 
التحديات و القرص صأمام القطاع الخاص لثنقديم

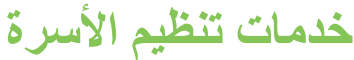

\section{أولاً: المنافسة من قبل القطاع الحكومي}

بعد القطاع الحكومي هو المنافس الأقوى للقطاع الخاص. فالأول يقدم

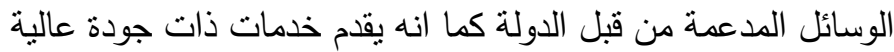

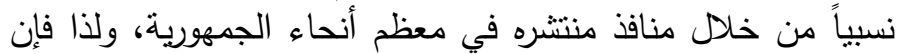

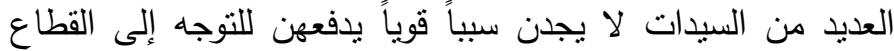

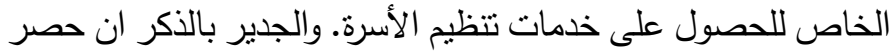

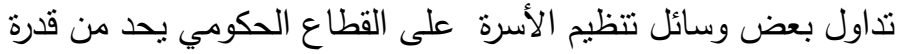

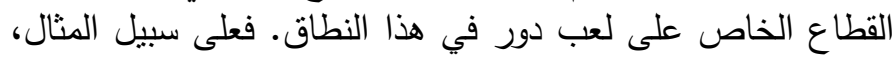
فإن وزارة الصحة والسكان هي المشتري والموزع الوحيد للحقن كل ثلاثنة

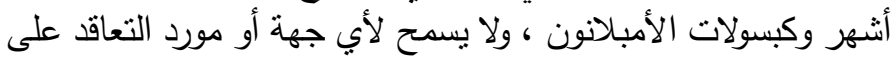
شراء "دييو بروفيرا" أو "إمبلانون" من الثركة التانة المصنعة أو تداولها.

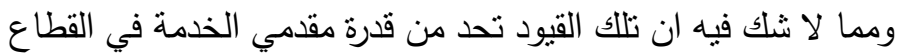

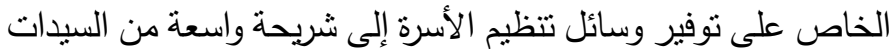
اللآتي لا برغبن أو لا يحتجن إلى استخدام القطاع الحكومي.

ومن ناحية أخرى فإن عدم وجود حوار أو تعاون كافي بين مؤسسات

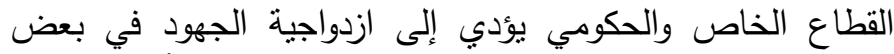

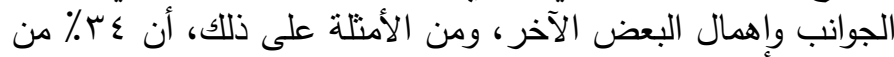

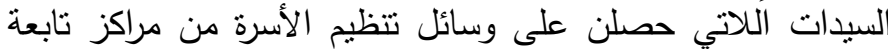

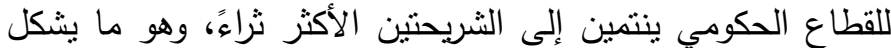

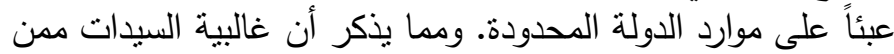

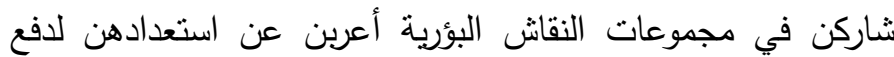

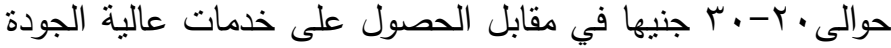

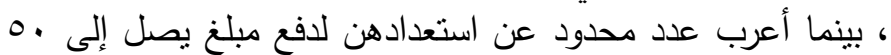

التوصية رقم

\section{اعتماد "نهج السوق الواحد"}

ينبغي لبرنامج تنظيم الأسرة في مصر اعتماد نهج

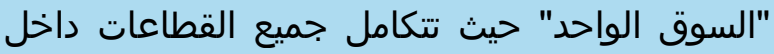

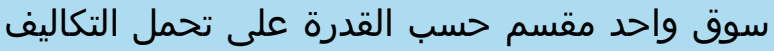

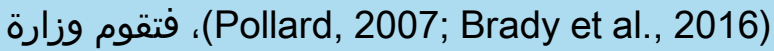
الصحة باستهداف السيدات في المناطق الريفية النائية

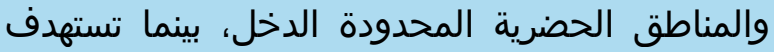
العيادات التابعة للجمعيات الأهلية السيدات المنات من الطبنة الطبقة

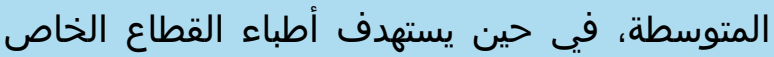
السيدات من الشرائح الاكثر ثراءً، أما الصيداء الصيدليات الخاص فتستهدف جميع الفئات من السيدات وكذلك الازواج.
العامة والاحصاء ؟ ( ـ ب) بين المناطق الريفية والحضرية، الا انها

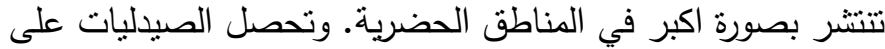

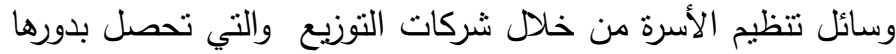

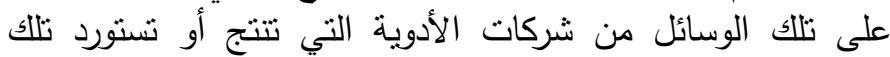

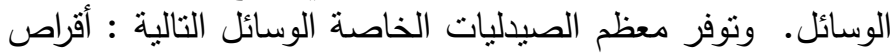
منع الحمل المركبة، أقراص الهرمون الواحد، الحقن الثهرية، والواقي الثراتي

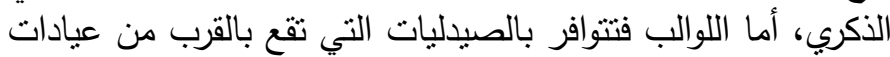

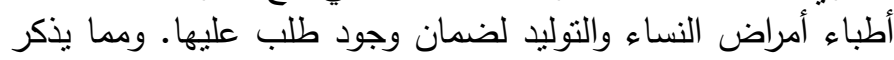
ان الحقن كل ثلاثة شهور وكبسولات الإمبلانون غير مسموح بتداولها

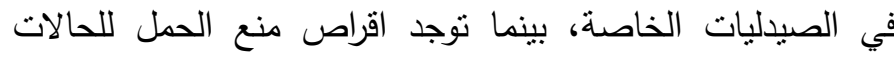

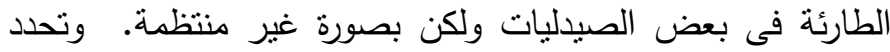
أسعار وسائل تتظيم الأسرة في الصيدليات وفقا للتسعيرة التي تحددها وزارة الصحة والسكان، إلا أنه يوجد تفاوت كبير السير في أسعار وسائل

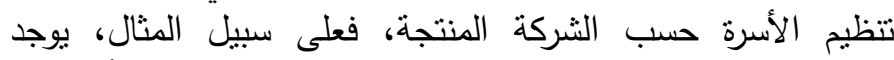

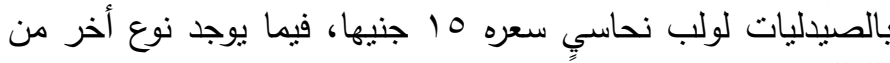

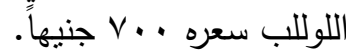

\section{9.}

"ابا كنت بشسري الحقنه من عند الدكتور الحصوصت ب كا جنية لكن

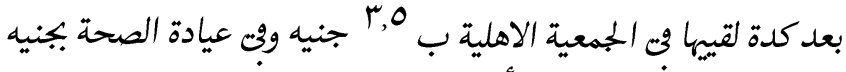

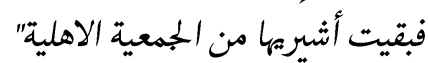

(احدى المشاركات" فتم مجموعات النقاش البؤرية)

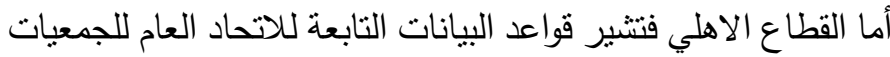

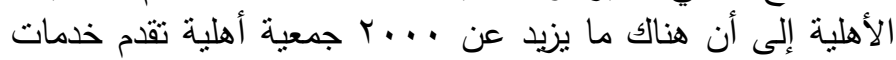

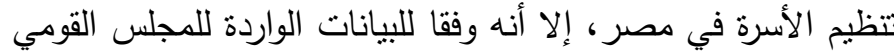

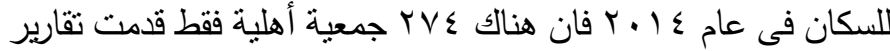

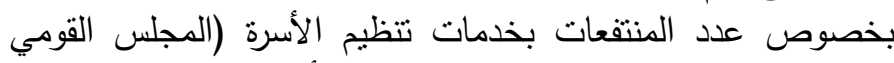

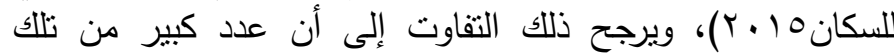

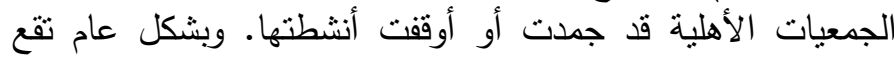

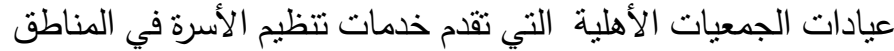

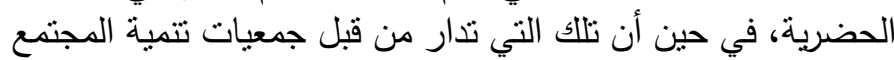
غالبا ما تقع في المناطق الريفية أو المناطق العشوائية. ومن الجئي الجمعيات

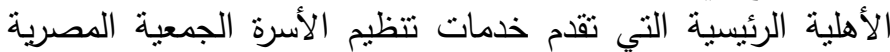

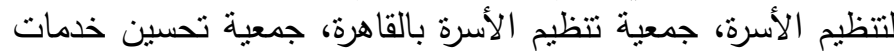
تتظيم الأسرة، والهيئة القبطية الإنجيلية للخدمات الألفرة الاجنماعية.

وتلبي عيادات الجمعيات الأهلية احتباجات السيدات من الطبقتين

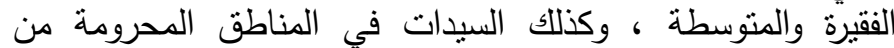
الخدمات الصحية، مثال المناطق العشوائية.

وتتلقى الجمعيات الأهلية وسائل تتظيم الأسرة المدعمة من وزارة الصحة الصحة الصها

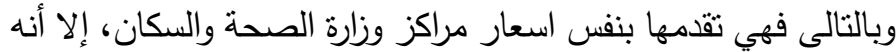

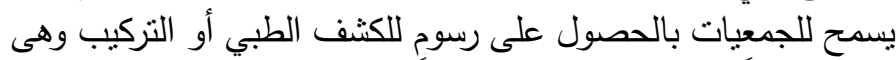

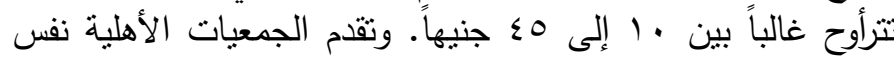

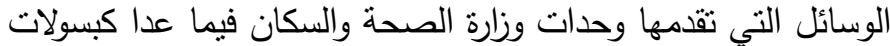
الأمبلانون التي لا يسمح للجمعيات الأهلية بتقديمها. 


$$
\text { التوصية رقم }
$$

ضمان اتاحة وسائل تنظيم الأسرة وتوفير

$$
\text { الخيار ات }
$$

على وزارة الصحة والسكان ان تعيد النظر في إجراءات

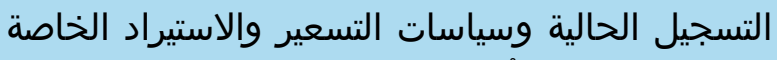

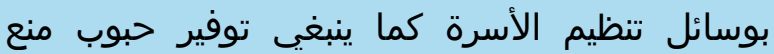

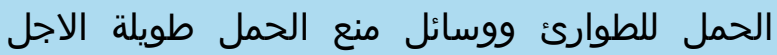

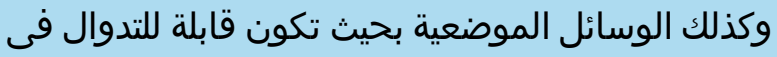

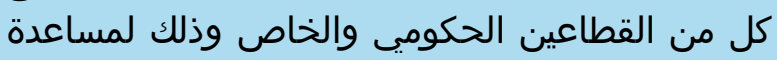

السيدات على تجنب مخاطر الحمل غير المخطط لهاص له.

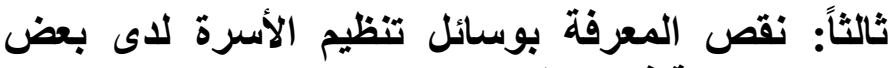

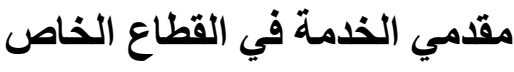

أثنار معظم الصيادلة الذين شاركوا في الدراسة الكيفية أنهم لم يتلقوا

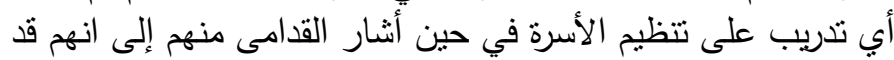

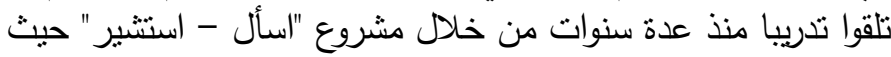

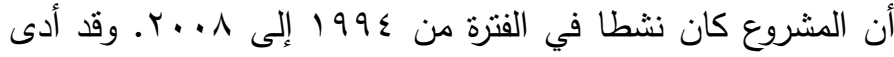

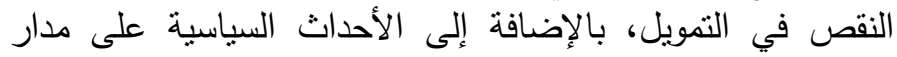

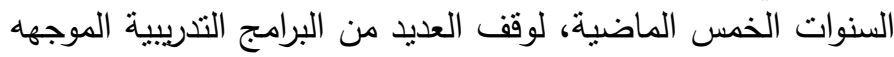

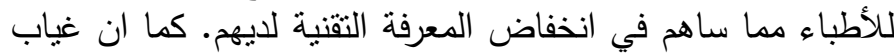

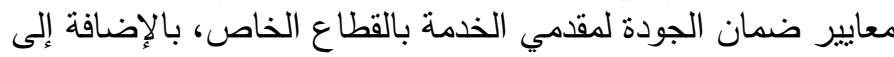

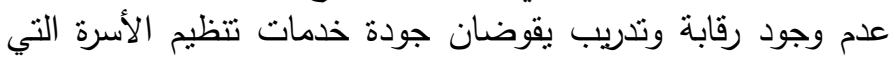
يقدمها القطاع الخاص.

وتتشير نتائج الدراسة الكيفية مع السيدات ومقدمي الخدمة إلى وجود

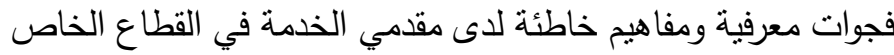

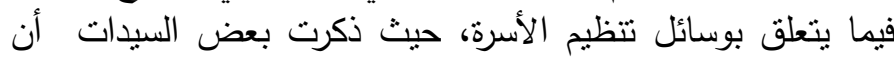

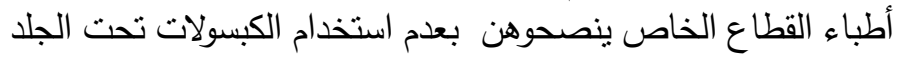

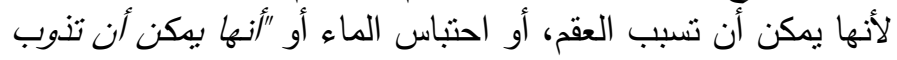

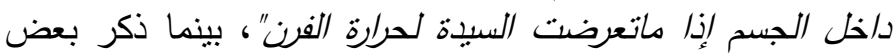

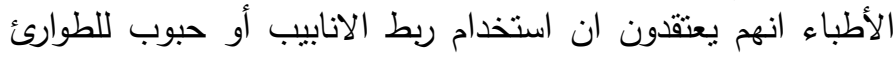

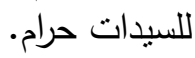

\section{9}

" اللت مش قادر اهمه ان يبيق اثنيس متجوزس جديد وعاوب يستخدموا

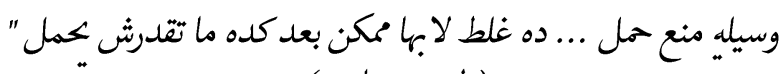

$$
\text { (طبيب خاص) }
$$

التوصية رقم

تعزيز الثراكات بين القطاعين الحكومي

يجب أن تكون العلاقة بين القطاعين الحكومي الحتبي

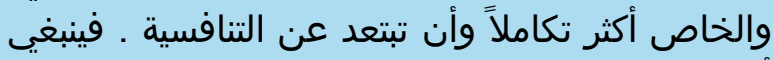

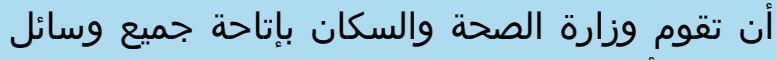
تنظيم الأسرة للقطاع الخاص (بما في ذلك الك الحقن الحن كل

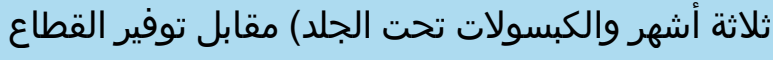

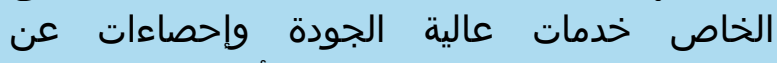

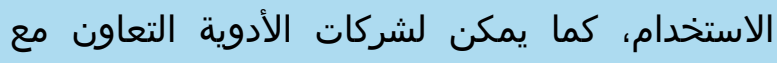

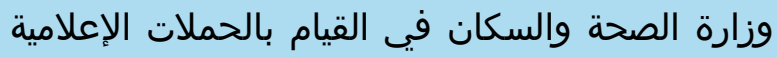

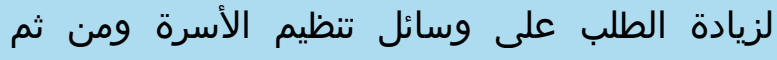
الاستفادة من بعض الطادي على الاعفاءات التيى تحصل عليم الاسرة ومن وزارة الصحة والسكان من القنوات التلفزيونيه الحكومية.

\section{ثانياً: الإجراءات البيروقراطية والسياسات التقييدية}

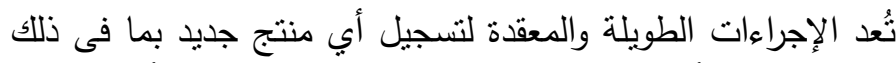

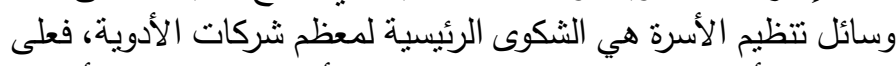

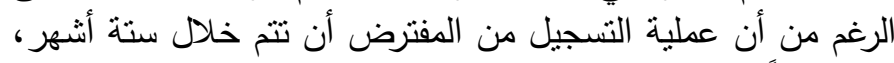

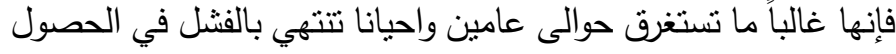

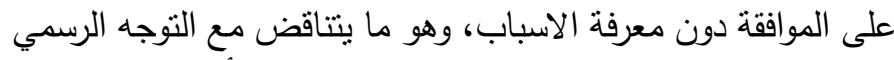

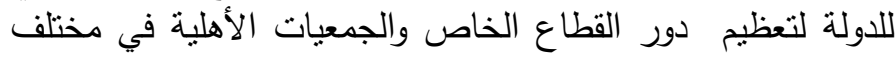
جوانب التتمية الاقتصادية والذي إنعكس في إستراتية وإنيجية التنمية

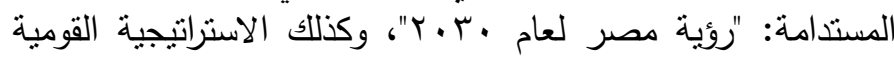

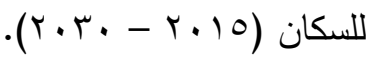

وقد أنشار بعض المشاركين إلى ان تسجيل العقاقير والمستحضرات

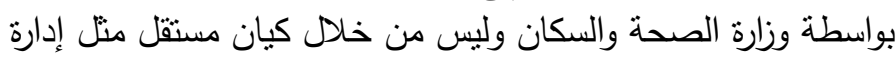

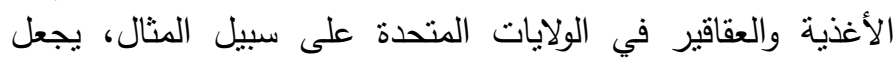
الاجراءات عرضه للتغيير المتكرر مع تغيير وزراء الصحة. الصني.

وفي نفس السياق اضاف المشاركون في الدراسة ان سياسات التسعير

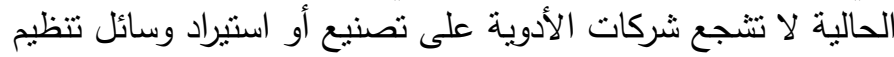

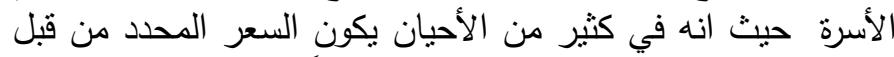

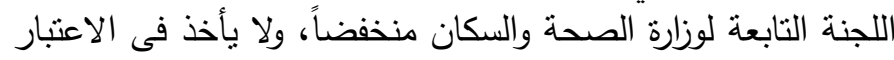

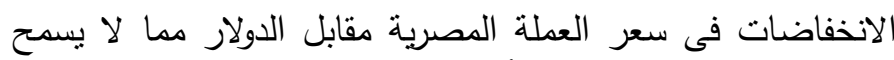

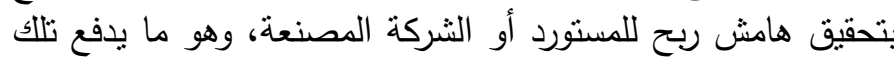

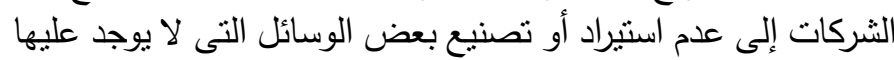

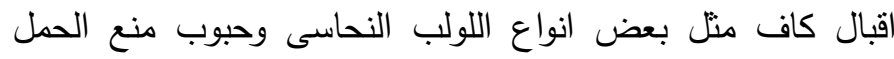

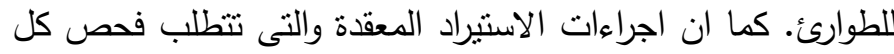

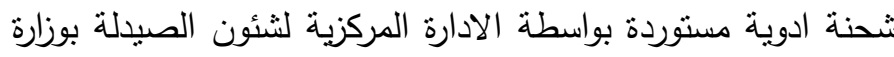

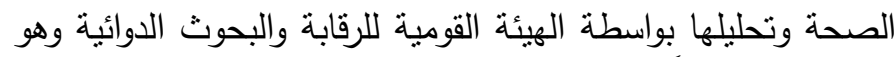

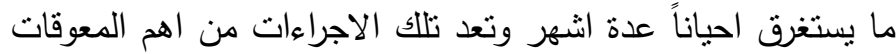

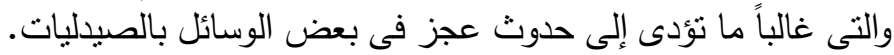

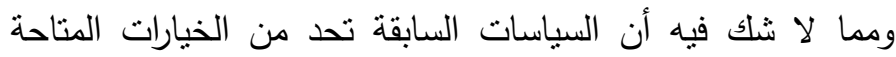
للسيدات وبالتالى تؤدي إلى عدم تلبية إحتياجاتهن. 
به \% ققط من السيدات اللاتى تترأوح أعمار هن بين ه 1 ـ ـ

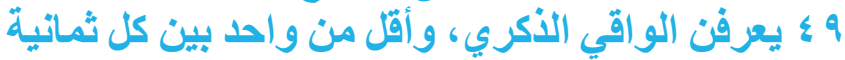
سيدات ورجال يعرفون عن حبوب الطوارئ فئ واهئ

.(EHIS, 2015)

$$
\text { التوصية رقم } 7
$$

$$
\text { استثمار الفرص لرفع الوعى بتنظيم الأسرة }
$$

ينبغي على أطباء القطاع الخاص استثمار الفرص

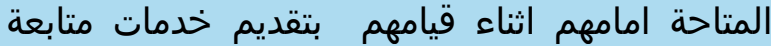
الحمل وما بعد الولادة والرعاية الصحية للطفل في في رفع

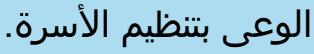

ومن الجدير بالذكر أن المعلومات الخاصة بوسائل تتظيم الأسرة في لئي

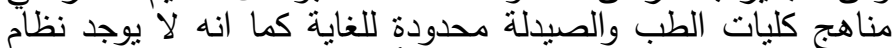

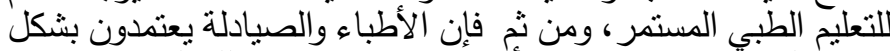

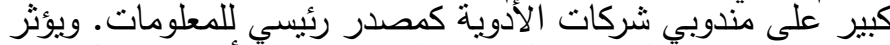

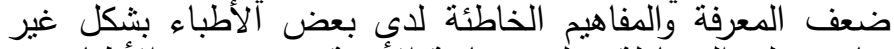

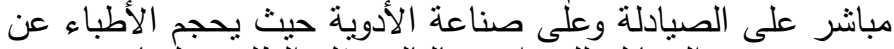

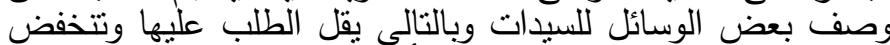
مبيعاتها مما يؤدي إلى وقق التتاجها أو استتيرادها.

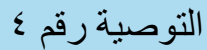

تدريب طلبة كليات الطب و الصيدلة على تنظيم

$$
\text { الأسرة }
$$

ينبغى ان تشتمل مناهج كليات الطب والصيدلة على معلومات موسعة عن تنظيم الأسرة، كما يناء ينبغ الأيى ان يحصل الأطباء المقيمون واطباء الأمتياز على تدريمات تلفيبيات عملية فى مجال تنظيم الاطباء المقيم والسباء الأمتياز على و تقديم المشورة وتركيب وخلع اللولب و كبسولات تحت الجلد.

$$
\text { التوصية رقم }
$$

رفع وعى الأطباء و الصيادلة بالتقنيات الحديثة الخاصة بتنظيم الأسرة الأر و

ينبغى على شركات الأدوية رفع الوعى لدى الأطباء

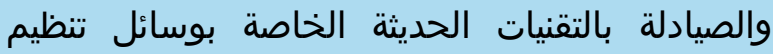
الأسرة من خلال الكتيبات والدوريات و والديات مندوبي شركات الأدوية وكذلك المؤتمرات وورش العمليات والدوريات مندو

\section{رابعاً: إنخفاض الطلب على خذمات القطاع الخاص}

شهدت الفترة من عام 1910 إلى عام ب . . ب نمواً كبيراً في انشطة

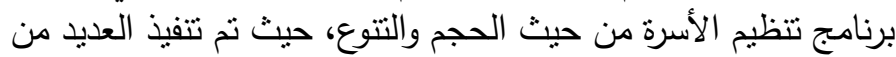

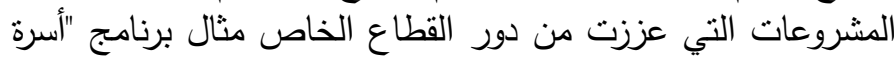

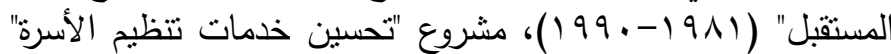

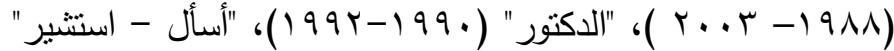

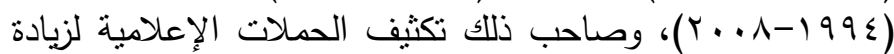

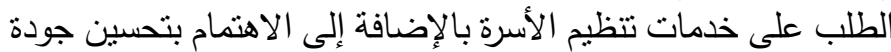

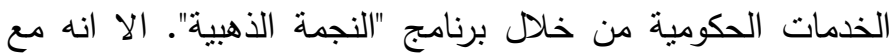

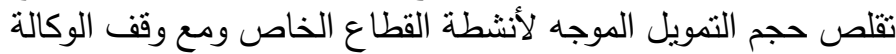

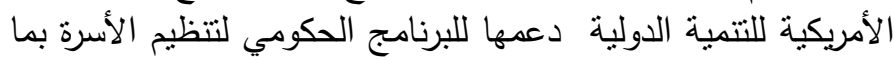

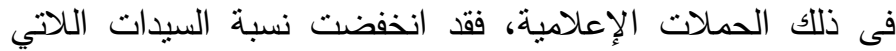

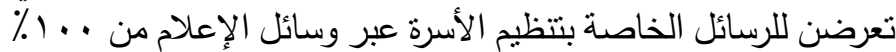

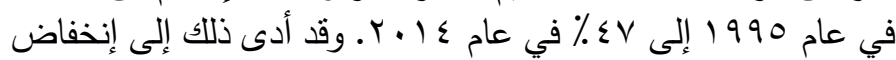

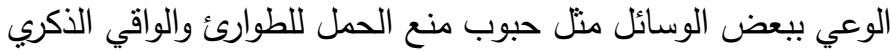
وهو ما يقلل من احتمالات الطلب عليها من قبل المنات المنتفعات أو وصفئ الوكيا

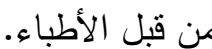


سادسا: تفرق الكيانات التابعة للقطاع الخاص

يتكون القطاع الخاص المعنى بتقديم خدمات تتظيم الأسرة من كيانات

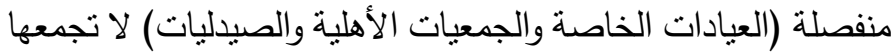

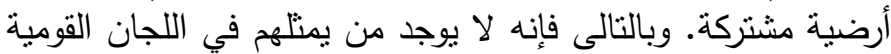

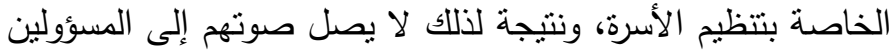

الحكومبين.

\section{9}

" القطاع الحاص ليس له نقابة أو جمعية للتشاور معها .... ولا يوجد مثثل

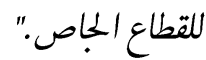

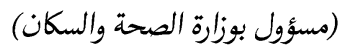

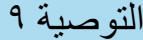

اقامة شبكة تضم مقدمى الخدمة بالقطاع الخاص

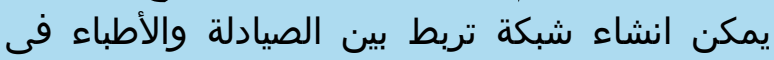
العيادات الخاصة (على سبيل المثال تفعيل شبكة "إسبال - استشير") بحيث تكون وسيلة للوصول إلى إلى كلى

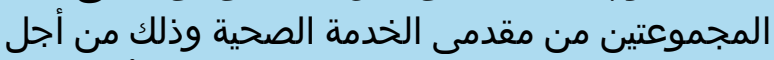

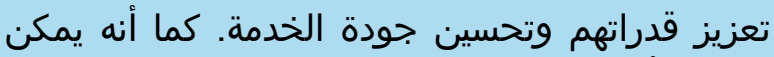

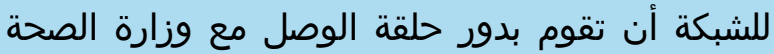

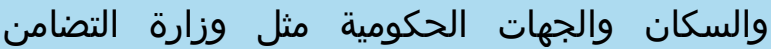
الاجتماعي ووزارة الصناعة والتجارة وغيرهم.

سوف يحتاج البرنامج القومي لتتظيم الأسرة في مصر في السنوات

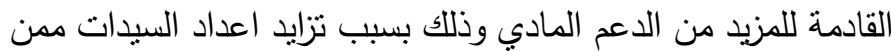

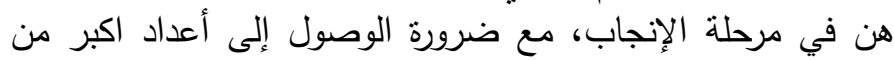

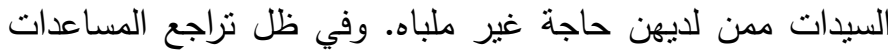
المقدمة من الجهات المانحة فإن ذلك يتطلب نعظيم الاستفادة من

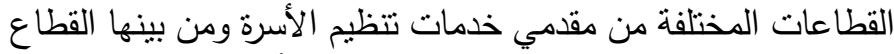

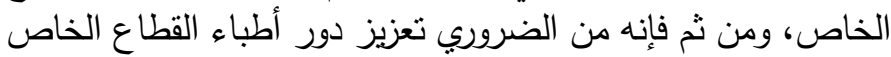

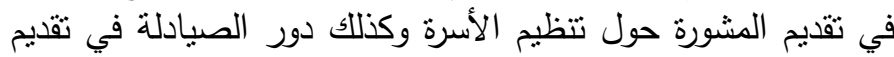

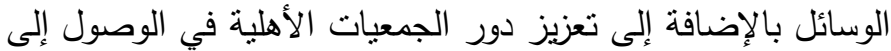
السيدات في المناطق المحرومة من الخدمة.

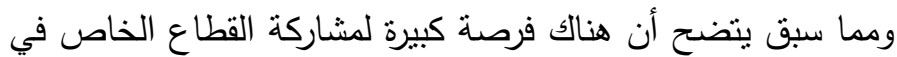

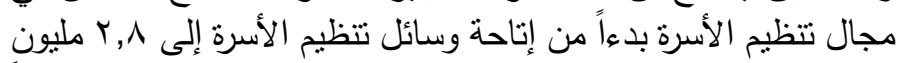

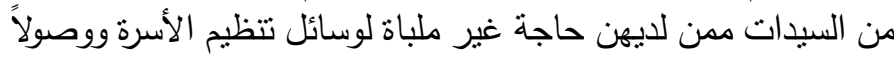

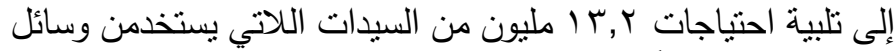

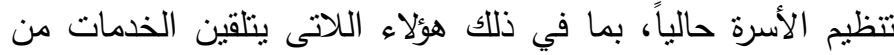

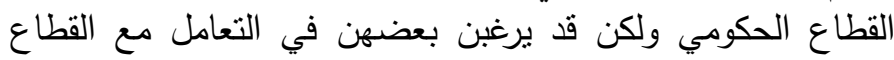

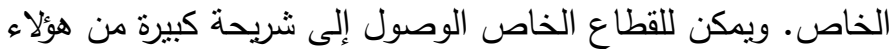
السيدات بشكل أفضل فى حالة وجود تعاون بين القطاعين القئ الحكومي

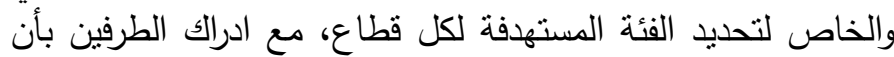

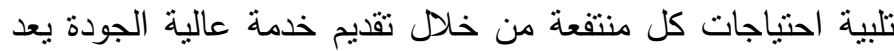

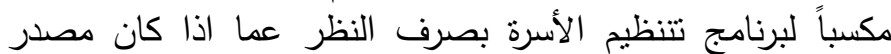

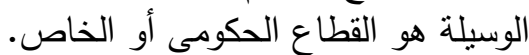

خامسا : معوقات نقص التمويل وتوفير الكوادر المدربه فى الجمعيات الأهلية

ويعد نقص التمويل هو التحدي الرئيسي الذي يواجه الجمعيات الأهلية،

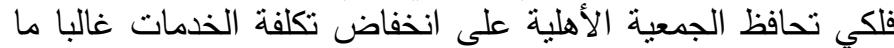

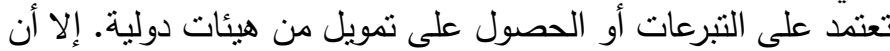

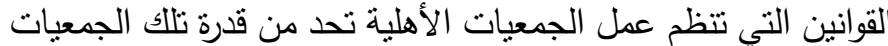

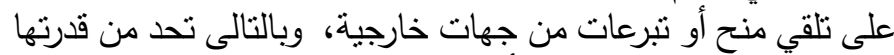

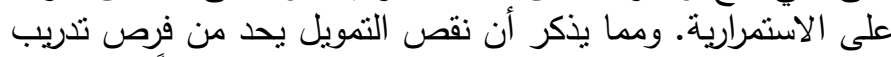

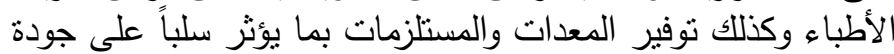

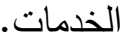

\section{انخفض عدد الجمعيات الأهلية التي تقدم خدمات تنظيم

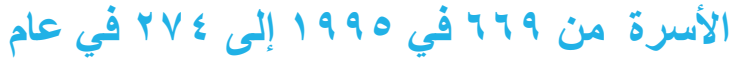

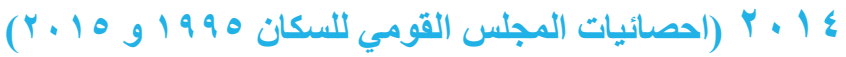

وفيما يخص التوظيف فان الجمعيات الأهلية غالباً ما تعتمد على الهى

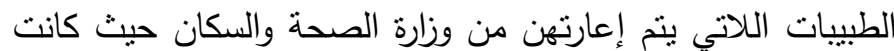

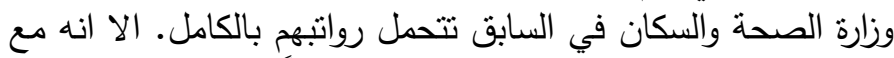
صدور قانون الخدمة المدنية الذي كان سارياً في الفترة من بوليو

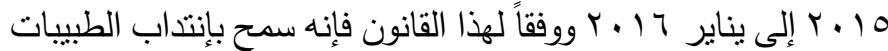

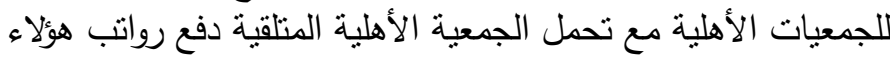

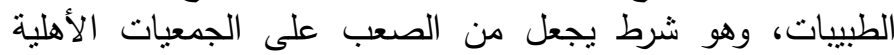

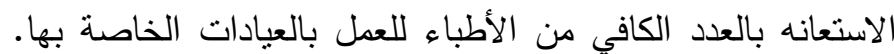

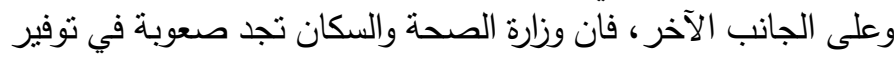

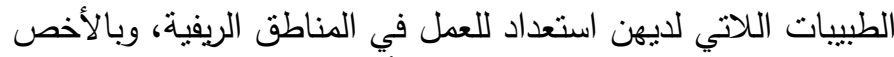

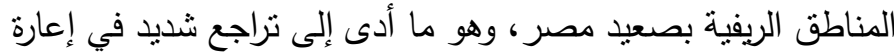
الأطباء إلى الجمعيات الأهلية.

\section{التوصية}

دعم وانشاء المزيد من منافذ القطاع الخاص

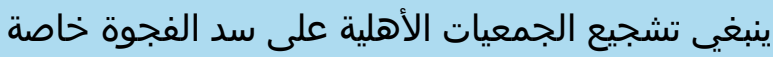

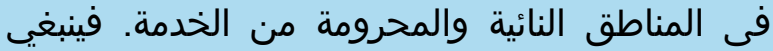
تبسيط وتسهيل الاجراءات الخاصة بتسجيل الجرات الجمعيلة الخدات وكذلك اجراءات الموافقة على حصول تلى تلك إت الجمعيات

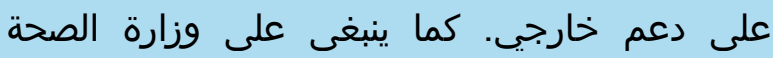

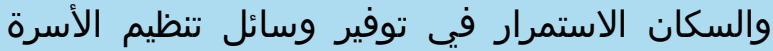

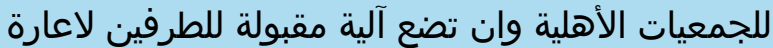

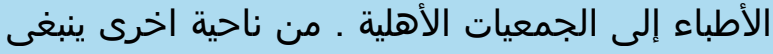

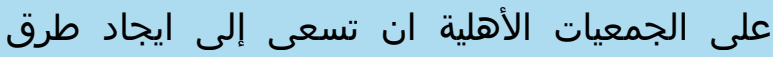

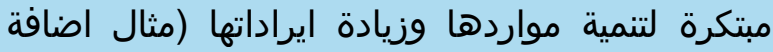

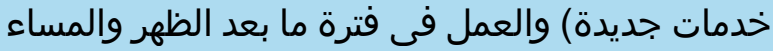
بحيث لا تتعارض ساعدات والعمل في فترة ما بعد مراكز وزارة الصحة والمساء

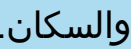


Brady C, Weeden L, Hutchings J., Parks, J. 2016. “Planning Guide for a Total Market Approach to Increase Access to Family Planning. Module 1: Landscape Assessment." Seattle, WA: PATH and Washington, DC: The Population Council, Evidence Project.

Cairo Demographic Center. 2002. Promoting the Role of the Private Sector as a Source of Family Planning Services and Products. Cairo: Cairo Demographic Center and Research Management Unit - National Population Council.

Central Agency for Public Mobilization and Statistics (CAPMAS). 2014. Census of Economic Establishments. Cairo: CAPMAS.

El-Zanaty F. and Ann Way. 2006. Egypt Demographic and Health Survey 2005. Cairo: Ministry of Health and Population, National Population Council, ElZanaty and Associates, and ORC Macro.

Fateem, E. and Wahba, M. 2012. "The Study of Reproductive Health Information, Attitudes and Practices of Bedouin Youth." Final Report. Cairo: The Egyptian Family Health Society.

Health Systems 20/20 Project. 2012. Assessment of Family Planning Program. Bethesda, MD: Health Systems 20/20 project, Abt Associates Inc.

\footnotetext{
نتقدم بالثكر لكل من ساهم في اجراء الدراسة واعداد التقرير الرئيسي كما نتقدم بالثكر إلى كل من سارة غطاس و طارق علم الدين لمساهمتهما في
} صياغة هذا الملخص.
Ministry of Health and Population, El-Zanaty and Associates and ICF Internation- al. 2015. Egypt Demographic and Health Survey 2014. Cairo, Egypt and Rock- ville, Maryland, USA: Ministry of Health and Population and ICF International.

Ministry of Health and Population, El-Zanaty and Associates, and ICF Interna- tional. 2015. Egypt Health Issues Survey 2015. Cairo, Egypt and Rockville, Maryland, USA: Ministry of Health and Population and ICF International.

Nakhimovsky, S. et al. 2011. Egypt National Health Accounts: 2008/09. Bethes- da, MD: Health Systems 20/20 project, Abt Associates Inc.

National Population Council. 1995. Annual Statistical Report on Family Planning Services. (In Arabic). Cairo: National Population Council.

National Population Council. 2015. Quarterly Statistical Report on Family Plan- ning Services (July - September 2015). (In Arabic). Cairo: National Population Council.

Pollard, R. 2007. "Social marketing: An introduction to the total market approach to commodities and services supply in low-income countries." Paper presented at the George Washington University Health Communication and Social Market- ing Symposium Series; Washington, DC.

Robinson W. and F. El-Zanaty. 2006. The Demographic Revolution in Modern Egypt.

$$
\text { تم استخلاص هذا الموجز من التقرير التالي: }
$$

Abdel-Tawab, N.; D. Oraby; B. Bellows. 2016. Situational Analysis of the Private Sector in the Delivery of Family Planning Services in Egypt: Current Status and Potential for Increased Involvement. Cairo: Population Council.
(USAID) ، تُفذ هذا المشروع بدعم من الوكاله الأمريكية للتنمية الدولية

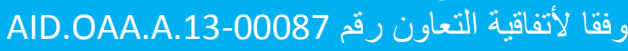

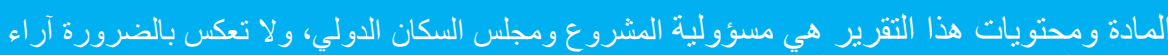

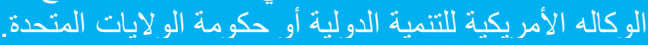

يستخدم مثروع Evidence منهج البحوث التطبيقبة لإستنباط وترجمة

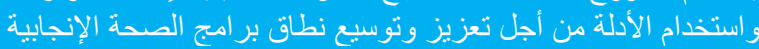

\section{Evidence}

وثنظيم الأسرة للحد من حالات الحمل غبر المخطط له حول العالم. وبر أس مشروع Evidence مجلس السكان

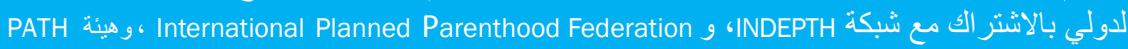
University Research Network و Population Reference Bureau

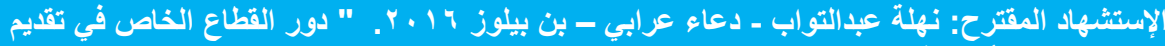

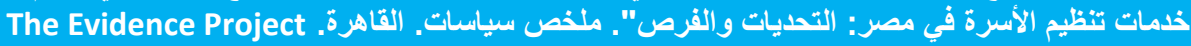

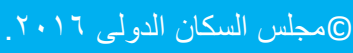

صورة الغلاف: Co 2003 Center for Communication Programs, Courtesy of Photoshare (C) 2002 Center for Communication Programs, Courtesy of Photoshare
THE EVIDENCE PROJECT

مجلس السكان الدولي

وه طريق مصر حلوان الزراعي الزي الئي

المعادي

صندوق بريد المعادي

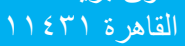

+r. r roro ०q7^:

evidenceproject@popcouncil.org 